\title{
Genome-wide long non-coding RNAs identified a panel of novel plasma biomarkers for gastric cancer diagnosis
}

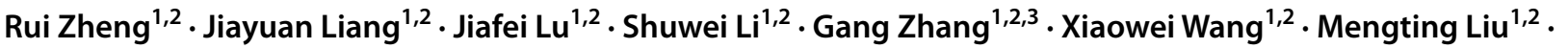 \\ Weizhi Wang ${ }^{4}$ Haiyan $\mathrm{Chu}^{1,2}$. Guoquan Tao ${ }^{5}$ Q Qinghong Zhao ${ }^{6}$ Meilin Wang ${ }^{1,2,7} \cdot$ Mulong Du $^{1,8} \cdot$ Fulin Qiang $^{9}$. \\ Zhengdong Zhang ${ }^{1,2,7}$
}

Received: 22 June 2018 / Accepted: 12 December 2018 / Published online: 2 January 2019

(c) The International Gastric Cancer Association and The Japanese Gastric Cancer Association 2019

\begin{abstract}
Background Although long non-coding RNAs (lncRNAs) are regarded as useful plasma-based biomarkers for cancer detection, the potential diagnostic value of IncRNAs in gastric cancer (GC) remains unclear.

Methods To screen promising lncRNAs biomarkers for GC, we performed genome-wide lncRNA microarray assay between five GC cases plasma and matched healthy controls plasma. The expression of candidate plasma-related lncRNAs were validated in two-phase validation of 446 subjects. The receiver operating characteristic curve was constructed for evaluating diagnostic accuracy. We also determined the origin and stability of plasma lncRNAs, and investigated biological effects of candidate lncRNAs on cellular phenotypes.

Results A total of 3878 lncRNAs were expressed differentially in GC plasma, among which the top 10 up-regulated lncRNAs were selected for further validation. A two-stage validation revealed that plasma levels of three lncRNAs (FAM49B-AS, GUSBP11, and CTDHUT) were significantly higher in GC plasma as compared with healthy controls $(P<0.05)$, and the combined area under curve of these lncRNAs was 0.818 (95\% CI 0.772-0.864). Moreover, these lncRNAs were stable and detectable in human plasma, and also enriched in extracellular fluid. The expression levels of all three lncRNAs dropped significantly on day 10 after radical surgery compared with preoperative levels $(P<0.05)$. Also, lncRNA FAM49B-AS significantly promoted GC cell viability and invasion.
\end{abstract}

Conclusions Plasma lncRNA FAM49B-AS, GUSBP11 and CTDHUT have a strong potential to serve as noninvasive biomarkers for GC diagnosis.

Keywords Plasma $\cdot$ LncRNA $\cdot$ Diagnosis $\cdot$ Biomarker $\cdot$ Gastric cancer

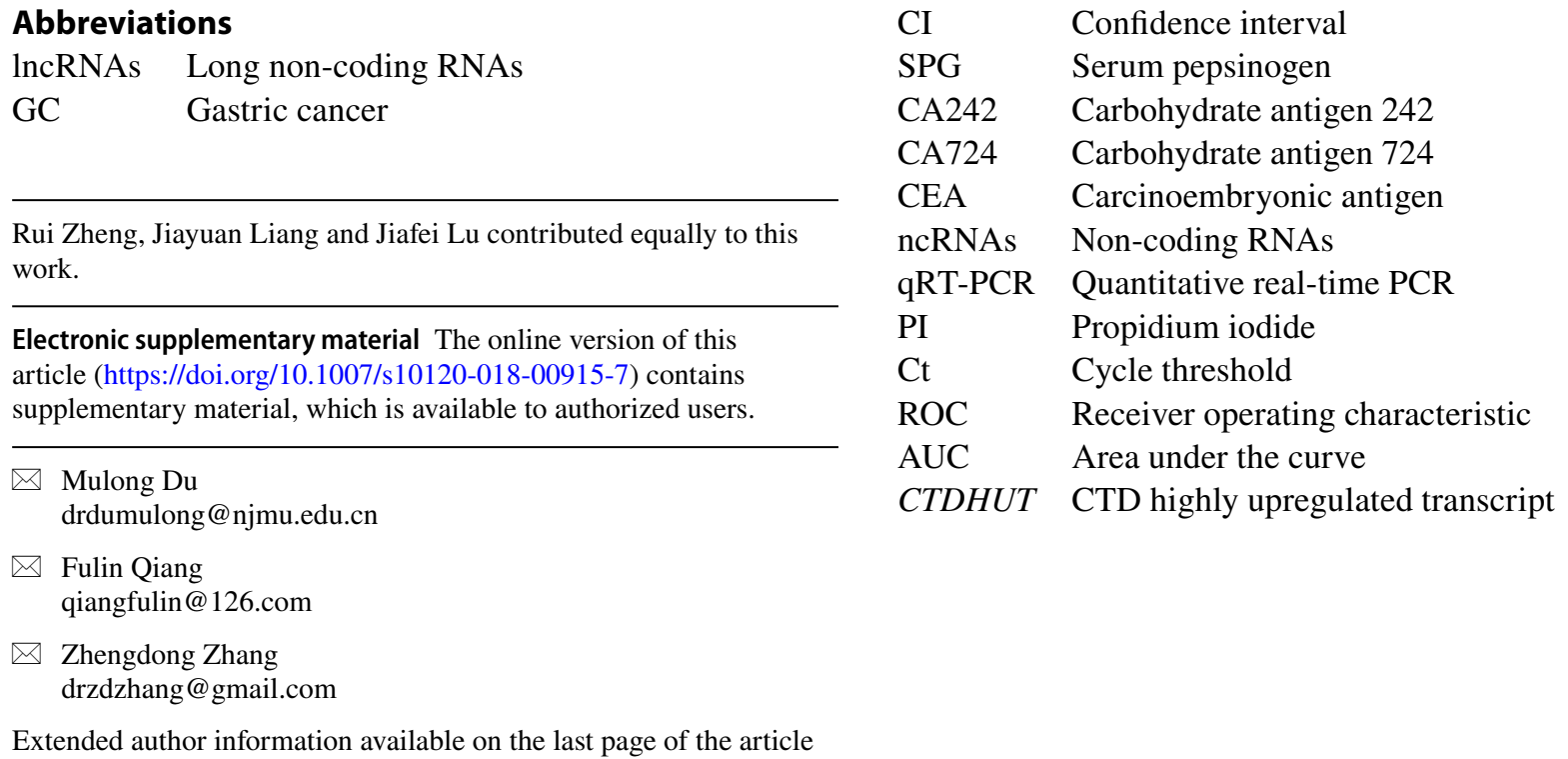

Abbreviations

lncRNAs Long non-coding RNAs

Gastric cancer

Extended author information available on the last page of the article 


\section{Introduction}

Gastric cancer (GC) is one of the most common malignancies worldwide, with a particularly high incidence rate in Eastern Asia [1, 2]. Although the decline trends in GC incidence and mortality have been reported in recent years, it remains the second most prevalent cancer in China, with approximately 679,100 new cases and 498,000 deaths in 2015 [3]. The 5-year overall survival rate was less than $25 \%$ in GC cases that were diagnosed at advanced stage with poor prognosis $[4,5]$. Biomarkers from the circulating system, including plasma and serum, provide a convenient and noninvasive method for diagnosis of tumor. However, current circulating tumor-specific biomarkers, such as serum pepsinogen (SPG), carbohydrate antigen (CA) 242, CA724 and carcinoembryonic antigen (CEA), have limited utility in GC diagnosis [6-9]. Identification of new biomarkers in blood for GC detection is essential.

Non-coding RNAs (ncRNAs) in plasma or serum, such as microRNA and long non-coding RNAs (lncRNAs), are novel noninvasive biomarkers for cancers [10-12]. The lncRNAs, longer than 200 nucleotides without proteincoding ability, play key roles in tumorigenesis, including influencing the proliferation, migration, invasion, metastasis, and apoptosis of tumor cells [13, 14]. Plasma lncRNA signatures are related to a variety of cancer types, such as prostate cancer [15], breast cancer [16], and esophageal squamous cell carcinoma [17].

Currently, a few studies have evaluated the diagnostic role of plasma lncRNAs in GC. Li and Pang et al. have identified that LINC00152 significantly expressed in GC tissues, and then could be extracted to plasma as a biomarker for predicting GC [18-20]. Meanwhile, Zhou et al. [21] selected IncRNA H19, that previously reported to be upregulated expression in GC, and demonstrated plasma lncRNA H19 may serve as a potential biomarkers for GC diagnosis. Furthermore, Liu et al. [22] reported a panel of plasma lncRNAs can be used as biomarkers for GC detection. Although these lncRNAs were detected by an IncRNA microarray assay in human plasma, the studies had the limited sample sizes in the validations and did not detect the biological effect of candidate lncRNAs. Therefore, the aim of the present study was to systematically explore potential plasma-derived lncRNA biomarkers for GC diagnosis.

\section{Methods}

\section{Study design and subjects}

This study was approved by the institutional review board of Nanjing Medical University. A total of 241 GC cases with histopathological confirmation were collected from the affiliated hospitals of Nanjing Medical University between 2006 and 2016, and 228 healthy controls were randomly recruited in the same hospitals during the same period. Among these subjects, five matched cases and controls were used to perform genome-wide lncRNA detection, and 13 cases were extracted peripheral blood before and 10 days after surgery. Meanwhile, we enrolled additional 37 subjects with benign gastric diseases, including gastritis, gastric polys, gastric erosion and gastric ulcer, as another control group.

\section{Genome-wide IncRNAs detection}

The Arraystar Human lncRNA Microarray v3.0 (Aksomics, China) containing approximately 30,586 human lncRNAs was used to analyze the whole genome-wide lncRNAs expression in plasma from five GC cases and matched healthy controls.

\section{RNA extraction and quantitative real-time PCR}

The total RNA was extracted from both plasma and culture media of cell lines using miRNeasy Serum/Plasma Kit (Qiagen, Germany). The total RNA from cell lines was processed using RNeasy Mini (Qiagen, Germany) following the manufacturer's instructions. A high-capacity cDNA reverse transcription kit (Invitrogen, USA) was used to generate cDNA. To evaluate the expression of candidate lncRNAs, quantitative real-time PCR (qRT-PCR) was performed using SYBR Green assays (Applied Biological Materials Inc, Canada) on an ABI 7900 system (Applied Biosystems, USA). The level of each candidate lncRNA was normalized to those of GAPDH and calculated with the formula $2^{-\Delta C_{\mathrm{t}}}$. The detection effect of IncRNAs was calculated using the detection rate [detection rate $=$ the number of samples $\left(C_{\mathrm{t}} \leq 35\right) /$ the total number of samples], by which higher detection rate indicated higher stability. The primer sequences are shown in Table S1. The expression of CA242 and CA724 in human plasma was detected using enzyme-linked immunosorbent assay (ELISA) kits (SenBeiJia Biotechnology Co., Ltd., China) according to the manufacturer's instructions.

\section{Cell lines and cell transfection}

Four human GC cell lines (MGC803, BGC823, SGC7901, and MKN28) and one normal human gastric (GES-1) cell 
line were used in this study. The cells were cultured in RPMI-DMEM medium (MMLTICELL, Canada) supplemented with $10 \%$ fetal bovine serum (Biological Industries, Israel). Cells were transfected with $F A M 49 B-A S$ overexpression plasmid/empty vector (NC) (Generay Biotech Co., Ltd., China) using Lipofectamine 2000 (Invitrogen, USA). The lentiviral vectors containing FAM49B-AS-siRNA1/controlsiRNA1 (si-NC) were synthesized by GeneChem (Shanghai, China).

\section{Cell proliferation assay}

After transfected with $F A M 49 B-A S$-siRNA1/si-NC lentiviral vectors or $F A M 49 B-A S$ overexpression/NC, $4.0 \times 10^{3}$ SGC cells and $5.0 \times 10^{3}$ MKN cells were seeded into 96-well plates and incubated for 7, 24, 48, and $72 \mathrm{~h}$. The cells were incubated with Cell Counting Kit-8 (Beyotime Biotechnology, China) for $2 \mathrm{~h}$ at $37^{\circ} \mathrm{C}$ and the absorbance was measured at $450 \mathrm{~nm}$ using the Infinite M200 spectrophotometer (Tecan, Switzerland).

\section{Cell invasion and migration assays}

The upper chamber of the trans-well (pore size, $8 \mu \mathrm{m}$; Millipore, USA) was spread by Matrigel (Millipore). After $24 \mathrm{~h}$ of incubation, $4.0 \times 10^{4}$ SGC7901 and MKN28 cells transfected with $F A M 49 B-A S$-siRNA1/si-NC lentiviral vectors and $F A M 49 B-A S$ overexpression/NC, respectively, were seeded into the upper chamber with $100 \mu \mathrm{l}$ of serum-free RPMI-DMEM medium, and RPMI-DMEM medium with $10 \%$ FBS was added to the lower well. Migration assay was similar to invasion assay except that the upper chamber was not coated with Matrigel. After incubation for $24 \mathrm{~h}$ at $37^{\circ} \mathrm{C}$ with $5 \% \mathrm{CO}_{2}$, cells on the lower chamber were fixed with 95\% methanol for 20 min and stained with $0.1 \%$ crystal violet for $15 \mathrm{~min}$. Then, five random fields were captured under 200× magnification inspection, and the numbers for cell invasion and migration in every field were quantified by ImageJ software.

\section{Flow-cytometric analysis for apoptosis and cell cycle}

To examine cell apoptosis, the transfected cells were stained with Annexin $\mathrm{V}$ and propidium iodide using Annexin V-FITC, and analyzed by flow cytometry. For the cell cycle analysis, the cells were fixed using $70 \%$ ethanol at $-20{ }^{\circ} \mathrm{C}$ for at least $18 \mathrm{~h}$, and stained with $500 \mu \mathrm{l}$ of PI for $15 \mathrm{~min}$.

\section{Statistical analysis}

Student's $t$ test or Pearson's $\chi^{2}$ test were performed using SAS version 9.4 software (SAS Institute, Inc., Cary, NC, USA) to document the differences between GC cases and healthy controls. The expression of each lncRNA was analyzed using comparative cycle threshold $\left(C_{\mathrm{t}}\right)$ on SDS 2.4 software (Applied Biosystems, Foster City, CA, USA). The specificity, sensitivity and area-under-the-curve (AUC) values for plasma lncRNAs were determined using the receiver operating characteristic (ROC) curve. $P$ values less than 0.05 were considered statistically significant.

\section{Results}

\section{Characteristics of subjects}

The characteristics of enrolled subjects are described in Table S2. For the two-phase validation subjects, there were no differences between GC cases and healthy controls in age and sex (all $P>0.05$ ). In the combined set, $48.6 \%$ and $51.4 \%$ of GC cases were in TNM stages I-II and III-IV, respectively.

\section{Differentially expressed IncRNAs from genome-wide IncRNA microarray assay}

An integral description of the study process is shown in Fig. 1. Genome-wide plasma lncRNA microarray analysis was conducted to detect differential lncRNA expression between five GC cases and matched healthy controls (Table S3). The heatmap and volcano-plot showed that of 3878 differentially expressed lncRNAs, 603 were upregulated and 3275 were downregulated (fold change $\geq 2$ and $P \leq 0.05$ ) (Fig. 2). Among these, we preferentially selected elevated lncRNAs; the top 10 up-regulated lncRNAs are listed in Table $\mathrm{S} 4$.

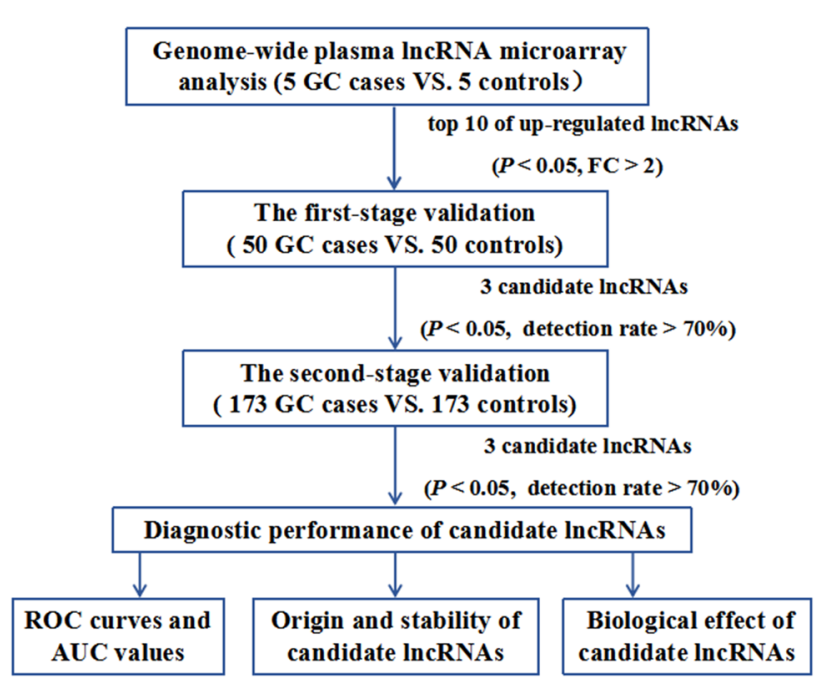

Fig. 1 Flow diagram of study design 
Fig. 2 Differentially expressed IncRNAs from genome-wide lncRNA microarray assay. a Heatmap result of microarray analysis between GC plasma and normal plasma samples. b Volcano plots of lncRNA expressions. The yellow plots were the differentially expressed lncRNAs with statistical significance (fold change $\geq 2$ and $P \leq 0.05)$ in GC plasma and normal plasma samples. $C$ case plasma, $N$ normal plasma
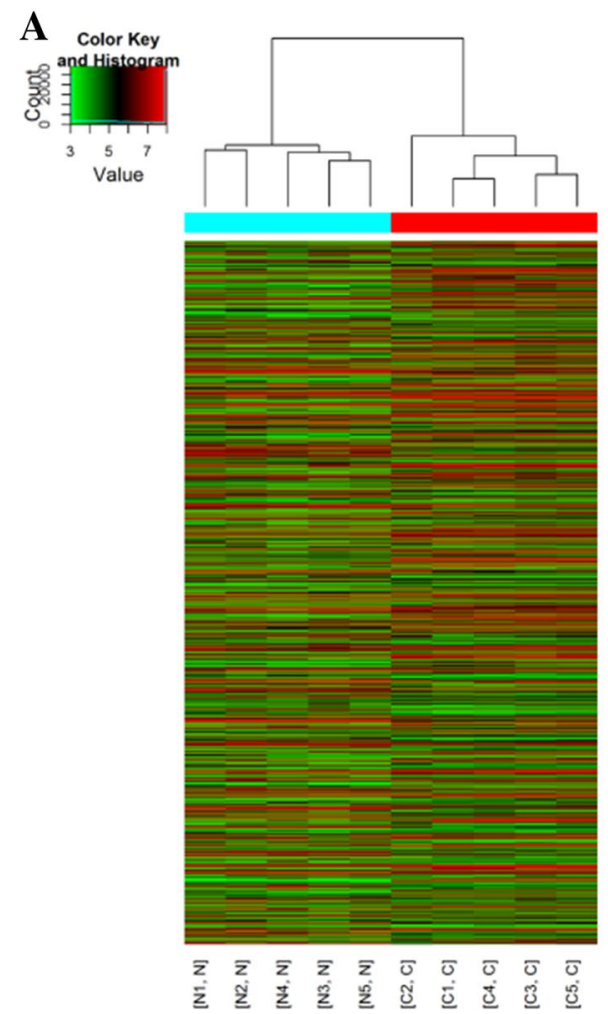

B

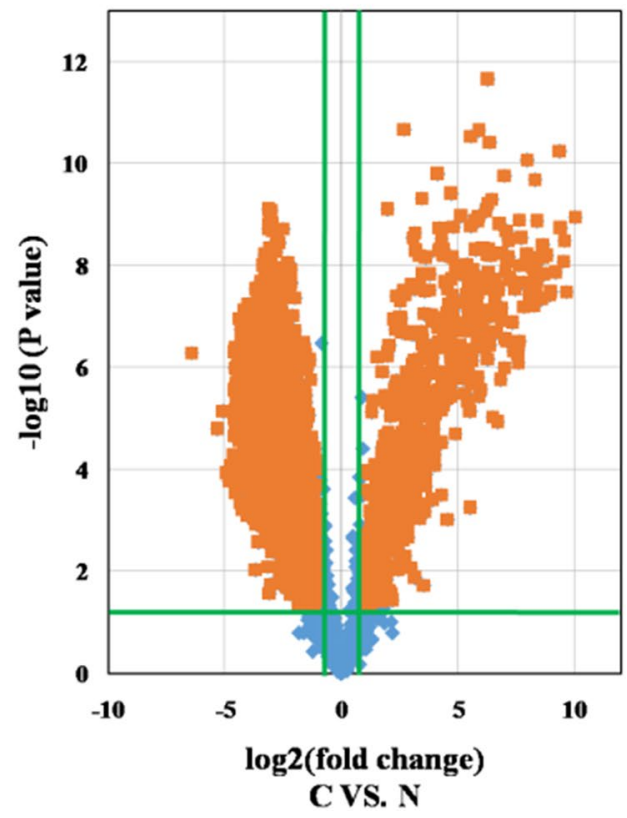

\section{Two independent validations of plasma IncRNAs}

To confirm the upregulated expression of the abovementioned 10 lncRNAs in plasma, we used qRT-PCR to examine their levels in a two-phase validation. GAPDH showed no significant differences between GC cases and normal controls in plasma (Figure S1A), indicating GAPDH is acceptable for normalizing plasma lncRNAs. In the first-stage validation, $50 \mathrm{GC}$ cases and 50 healthy controls were recruited to determine the above 10 candidate lncRNAs. As shown in Fig. 3a, b, lncRNA FAM49B$A S$ (ENST00000524100), GUSBP11 (uc002zxm.3) and CTD highly upregulated transcript (CTDHUT) (ENST00000446853) displayed significantly higher levels in GC cases (all $P<0.05$ and detection rate $>70 \%$ ). Subsequently, a total of 173 GC cases and 173 healthy controls were enrolled in the second validation. In line with the first-stage validation, FAM49B-AS, GUSBP11, and $C T D H U T$ were significantly upregulated in $\mathrm{GC}$ cases compared with healthy controls (all $P<0.05$ and detection rate $>70 \%$; Fig. $3 \mathrm{c}-\mathrm{f}$ ). Moreover, we evaluated the expression pattern of three candidate IncRNAs in subjects with benign gastric diseases (Table S5). As illustrated in Figure S1B, GUSBP11 levels were significantly upregulated in subjects with benign gastric diseases compared with controls, whereas FAM49B-AS and CTDHUT showed increasing trend levels from controls to subjects with benign gastric diseases. Furthermore, no significant association was observed between each lncRNA level and their clinical features (Figure S1C).

\section{Evaluation of the diagnostic performance of plasma IncRNAs}

Based on the above observations, we combined all the subjects in a two-stage validation to determine the sensitivity and specificity of the three plasma lncRNAs by generating ROC curves and analyzing AUC. The AUC values for plasma FAM49B-AS, GUSBP11, and CTDHUT for GC cases vs. healthy controls were 0.609 (95\% CI 0.548-0.670), 0.635 (95\% CI 0.575-0.695) and 0.762 (95\% CI 0.711-0.813), respectively. However, when three lncRNAs were detected in combination, the AUC was increased to 0.818 (95\% CI $0.772-0.864$ ), and sensitivity and specificity were raised to $77.5 \%$ and $73.9 \%$, respectively (Fig. $4 a$ ). We then combined the traditional clinical biomarkers of CA242 or CA724 and these three lncRNAs, and found that the combination of CA242 and CA724 yielded an AUC of 0.784 (95\% CI $0.734-0.835$ ) and 0.943 (95\% CI 0.919-0.968), respectively, which were significantly increased compared with individual CA242 (AUC $=0.658$ ) or CA724 (AUC $=0.911)$. Additionally, when integrating three IncRNAs, CA242, and CA724, they displayed the best diagnostic performance with AUC of 0.952 (95\% CI $0.931-0.974)$, sensitivity of $93.2 \%$ and specificity of $86.6 \%$ (Figure S1D). 
Fig. 3 Two independent validations of plasma lncRNAs. a The detection rate of $10 \mathrm{lncRNAs}$ between cases and controls in the first-stage validation. $\mathbf{b}$ qRTPCR detection of 10 lncRNAs expression in the first-stage validation. $\mathbf{c}$ The detection rate of FAM49B-AS, GUSBP11, and $C T D H U T$ between cases and controls in the second-stage validation. d-f Distribution of $F A M 49 B-A S$, GUSBP11, and $C T D H U T$ expressions in the plasma from cases and controls in the second-stage validation. The plasma lncRNAs expressions were normalized to GAPDH. Results are presented as mean \pm SD. $* P<0.05$
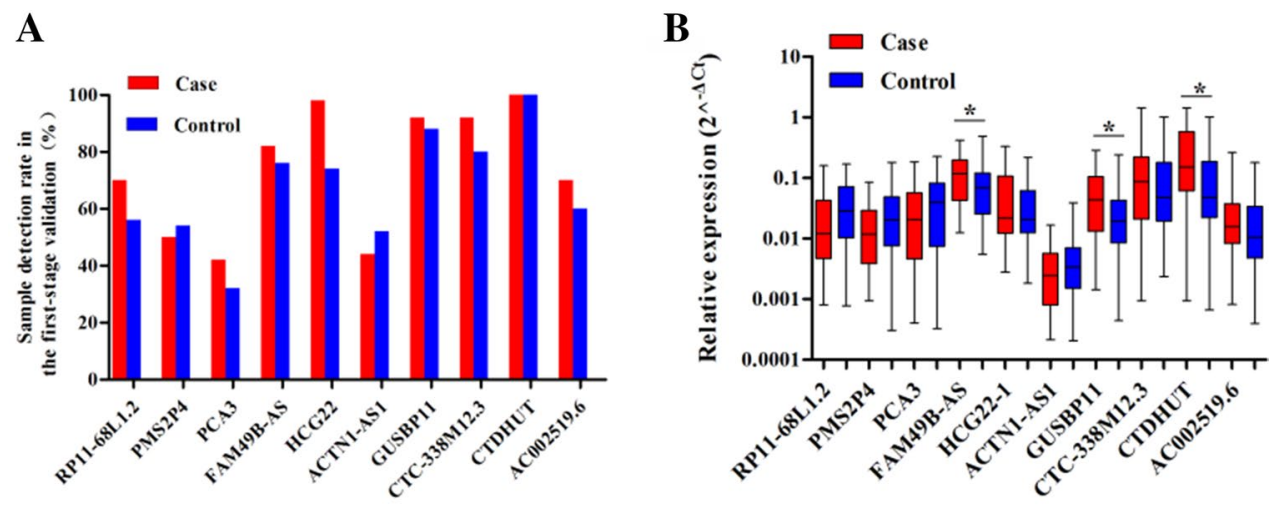

C

D

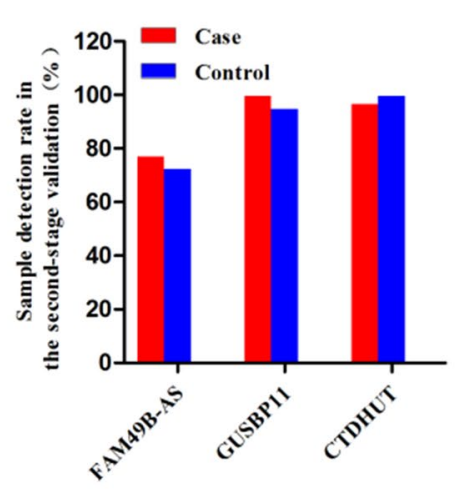

E

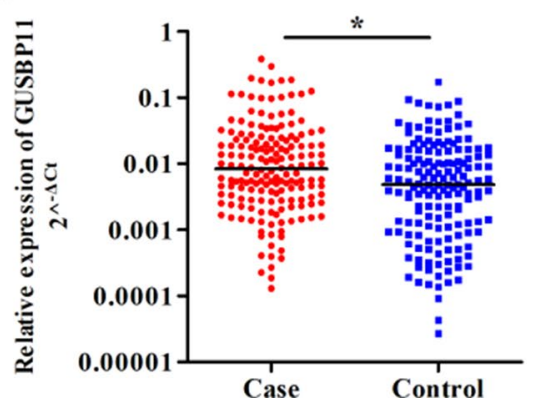

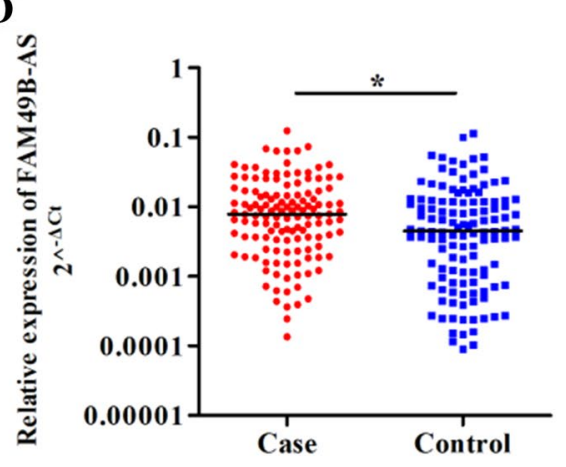

F

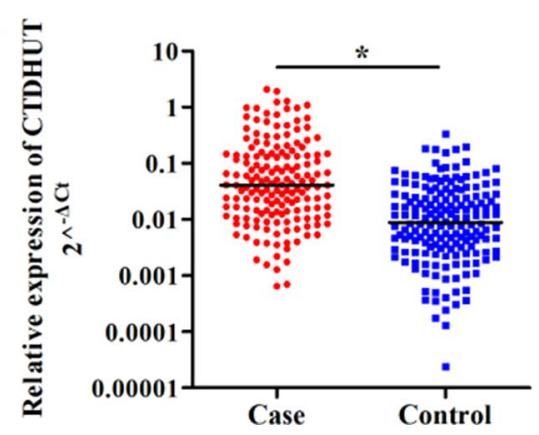

Based on the results in the two-stage validation, we assessed the origin of three candidate lncRNAs. We first measured the expression levels of these three plasma lncRNAs by qRT-PCR in 13 cases before surgery (pre) and 10 days after radical gastrectomy (post), which $53.8 \%$ cases were in stage III/IV and $53.8 \%$ cases with intestinal-type of GC (Table S6). The results showed that the expression of each lncRNA declined sharply in GC cases on day 10 after surgery $(P<0.05$; Fig. $4 \mathrm{~b})$. We then investigated the expression of these three IncRNAs in 13 paired preoperative/postoperative cases and matched healthy controls. After radical gastrectomy, the expression of $F A M 49 B-A S$ was in accordance with that in healthy controls $(P>0.05$; Figure S2A). In addition, the expression of three lncRNAs was measured in the corresponding culture medium of four GC cell lines (MGC803, BGC823, SGC7901, and MKN28) and
GES-1. Compared with the culture medium of GES-1, the expression of three lncRNAs significantly increased in the corresponding culture medium of four GC cell lines after 24/48 $\mathrm{h}$ incubation (Figure S2B-D). These results indicated that both tumor cell lines and GES-1 could secrete these three IncRNAs, especially in GC cell lines. Next, we measured the above-mentioned three lncRNA levels in BGC823, SGC7901, and GES-1 cells, and compared the expression of three lncRNAs in the corresponding culture medium after incubation for $24 \mathrm{~h}$. Consistent with the results from the culture medium of GC cell lines, FAM49B-AS, GUSBP11, and $C T D H U T$ also showed significantly elevated expression in BGC823 and SGC7901 cell lines compared with GES-1, confirming their GC-derived origin (Fig. 4c).

To investigate the stability of three lncRNAs in plasma, we kept the plasma samples under harsh conditions such as 
A

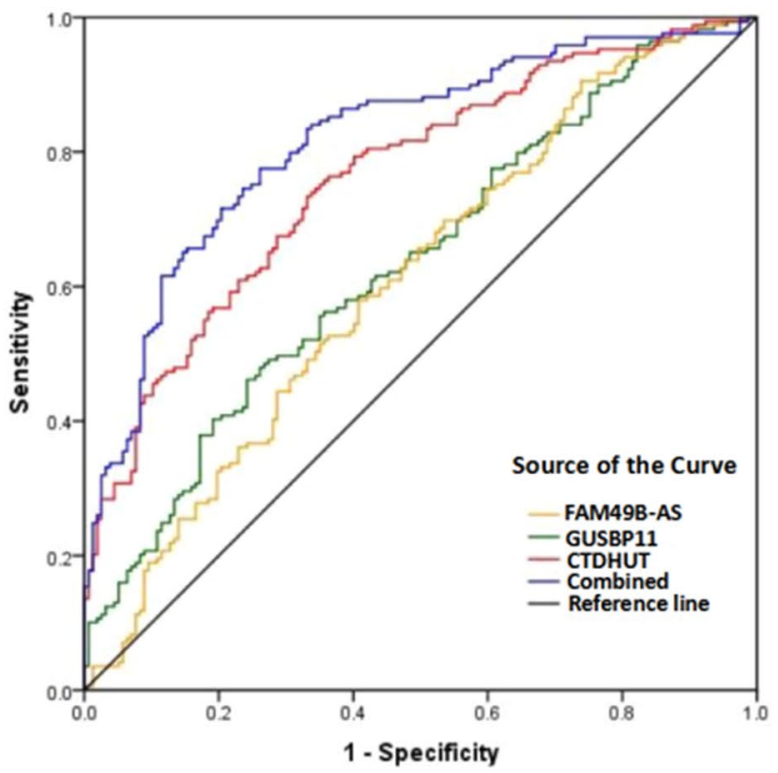

B
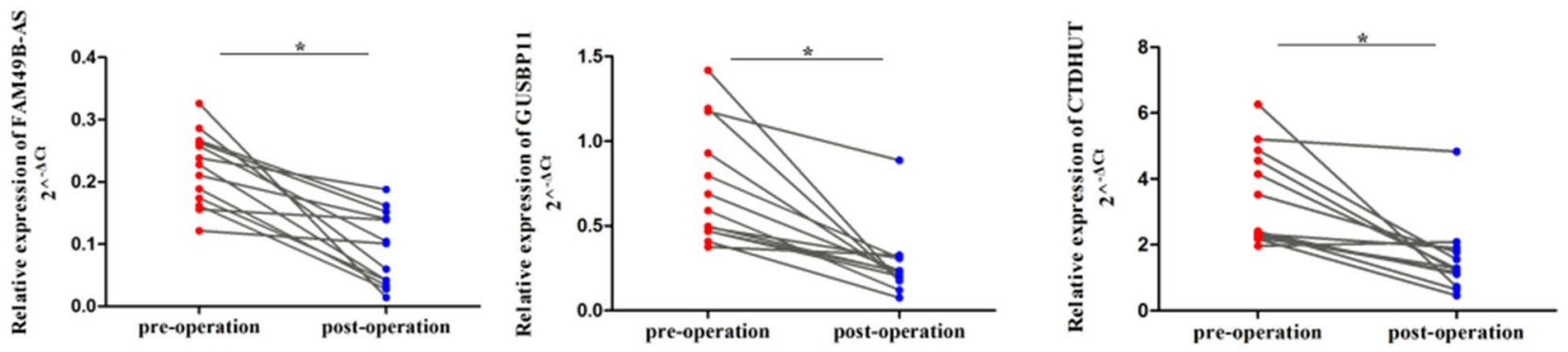

C
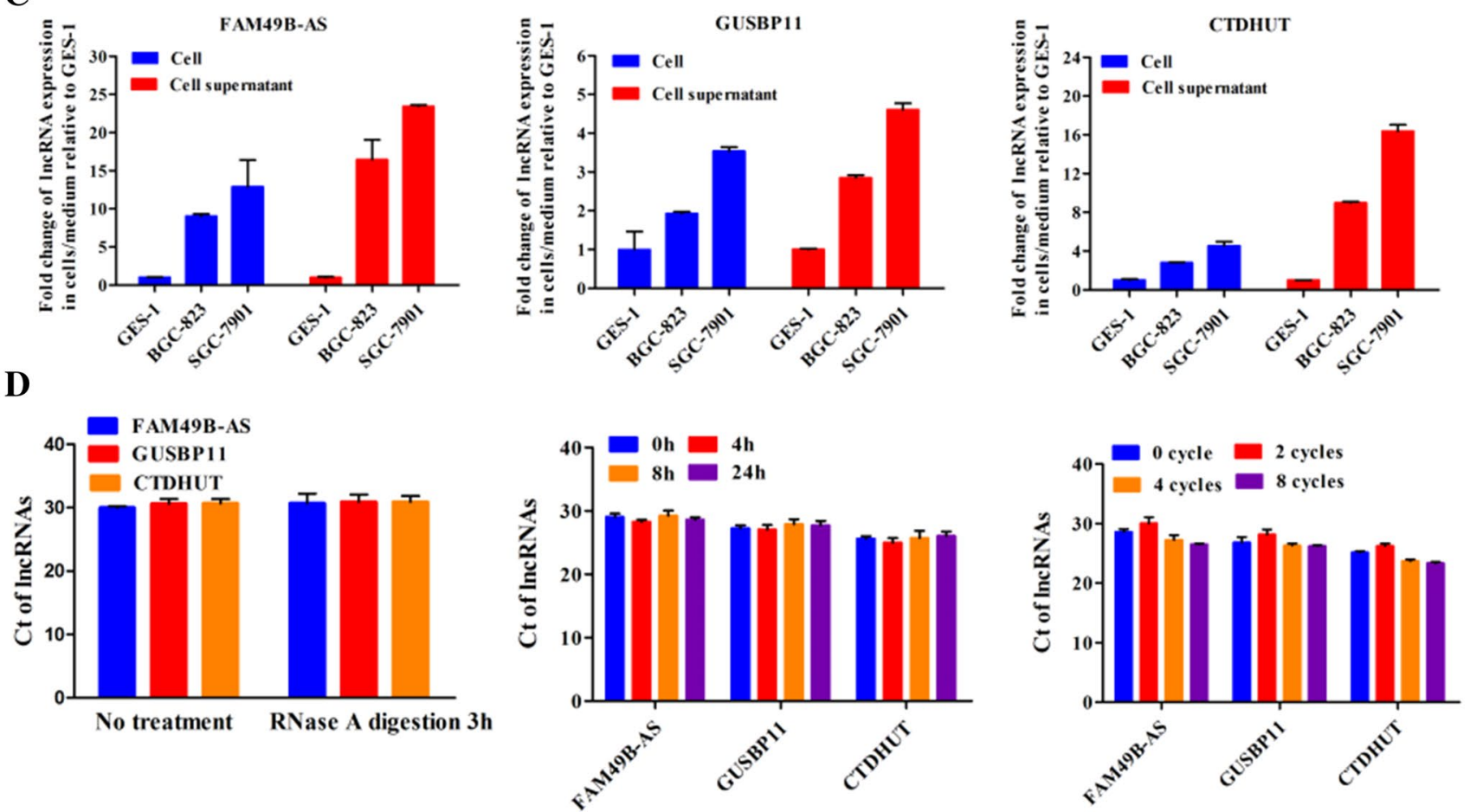
४Fig. 4 Evaluation of the diagnostic performance of plasma lncRNAs. a The ROCs of the three plasma lncRNAs in combined set. b qRTPCR detection of FAM49B-AS, GUSBP11, and CTDHUT expressions on preoperative (pre-operation)/postoperative (post-operation) patients with GC. c qRT-PCR detection of FAM49B-AS, GUSBP11, and CTDHUT expressions in cell lines (BGC823, SGC7901 and GES-1) and corresponding culture medium for $24 \mathrm{~h}$. d Stability of plasma lncRNAs was detected under different conditions: RNase A digestion for $3 \mathrm{~h}$, incubation at room temperature $0 \mathrm{~h}, 4 \mathrm{~h}, 8 \mathrm{~h}$, and $24 \mathrm{~h}$, and repeated $0,2,4$, and 8 freeze-thaw cycles. Results are presented as mean $\pm \mathrm{SD}$. $* P<0.05$

RNase A digestion for $3 \mathrm{~h}$, incubation at room temperature for $0,4,8$, and $24 \mathrm{~h}$, and repeated $0,2,4$ and 8 freeze-thaw cycles. Results indicated that the concentrations of these three plasma lncRNAs were hardly altered in such treatment (Fig. 4d).

\section{Effect of plasma IncRNAs on GC cellular phenotypes}

Understanding the function of plasma lncRNAs in tumorigenesis would facilitate their clinical application. The potential coding ability [23] (http://cpc.cbi.pku.edu.cn/ and http:// lilab.research.bcm.edu) and transcript properties that take into account whether transcripts with known non-coding RNA transcripts (http://genome-asia.ucsc.edu) were used to predict the biological functional of three candidate lncRNAs. Because $F A M 49 B$ - $A S$ has a very low coding potential, we preferentially selected it to observe whether it could promote biological malignant behavior of GC cells.

We investigated the expression of $F A M 49 B-A S$ in the four GC cell lines, and found that the expression of FAM49B$A S$ was abundant in SGC7901 cells, whereas the lowest in MKN28 cells (Fig. 5a). To further detect the function role of $F A M 49 B-A S$, we used siRNAs/NC lentiviral vector of $F A M 49 B$ - $A S$ to treat SGC7901 cells, and overexpression/NC of FAM49B-AS to treat MKN28 cells. FAM49B$A S$ levels were significantly decreased in SGC7901 cells after stable knockdown of $F A M 49 B-A S$, and significantly increased in MKN28 after overexpression of $F A M 49 B-A S$ (Fig. 5b). Then, a CCK8 assay revealed that proliferation of SGC7901 cells was remarkably impaired after FAM49B$A S$ stable knockdown for $24 \mathrm{~h}, 48 \mathrm{~h}$, and $72 \mathrm{~h}$. By contrast, FAM49B-AS overexpression in MKN28 significantly promoted cell proliferation (Fig. 5c). Compared with the NC group, SGC7901 cells with downregulation of FAM49B-AS showed significantly reduced invasion abilities, whereas MKN28 with the up-regulation of $F A M 49 B-A S$ significantly increased invasion abilities (Fig. 5d). Also, FAM49B-AS arrested the SGC7901 cell cycles at the G1 and G2 phase, and showed a trend toward arresting MKN28 cell cycles at the G1 phase (Fig. 5e). However, we did not find significant differentials in cellular migration and apoptosis affected by FAM49B-AS (Figure S3A-C).

\section{Discussion}

The majority of GC cases are diagnosed as an advanced stage, indicating an urgent need for promising biomarkers in GC diagnosis [24, 25]. Because lncRNAs can be detected in plasma samples in a highly stable form, and are considered to be effective noninvasive biomarkers for cancer diagnosis [11, 13, 26, 27]. Meanwhile, previous studies focused on the diagnostic performance of preselected lncRNAs, leaving a large number of lncRNAs unexplored [9]. In this study, we therefore screened promising lncRNA biomarkers in GC by a genome-wide lncRNA screening strategy between GC cases plasma and matched controls plasma. Based on this array result, we first identified that FAM49B-AS, GUSBP11, and CTDHUT levels were statistically upregulated in GC cases compared to healthy controls in a two-phase validation. Especially, we also elucidated that above-mentioned three lncRNAs could represent novel biomarkers for diagnosis of GC by examining their detection rate, stability, tumor-derived origin, and biological malignant behavior.

Correct endogenous controls ensure accurate detection of plasma lncRNA levels. We confirmed that plasma GAPDH levels are not affected in cases and controls using cel-miR-39 as an exogenous control, which is consistent with previous studies $[18,28]$. In the first-stage validation, the results showed that FAM49B-AS, GUSBP11 and $C T D H U T$ levels were significantly elevated in GC cases compared with healthy controls. We further detected the expression of these three IncRNAs in the first-stage validation and subjects with benign gastric diseases. Compared with controls, GUSBP11 significantly increased in subjects with benign gastric diseases; however, FAM49B-AS and $C T D H U T$ showed a trend toward elevating expression in group of benign gastric diseases, which could be due to small sample size. However, GUSBP11 levels were decreased in cases compared with subjects with benign gastric diseases. These findings suggest that further largescale population studies are needed to evaluate these lncRNA levels in subjects with benign gastric diseases. In the second validation, we found that $F A M 49 B-A S$, GUSBP11, and CTDHUT dramatically increased in GC plasma compared with healthy controls, consistent with our results in the first-stage validation. Next, we combined all of the subjects from the two-phase validation, and found that the combination of these three lncRNAs yielded more effective diagnoses of GC than the use of lncRNAs individually. However, no significant associations were observed between each candidate lncRNA and GC characteristics, suggesting further validation with large samples is warranted. Furthermore, we found that the combination of CA242, CA724 and three lncRNAs 

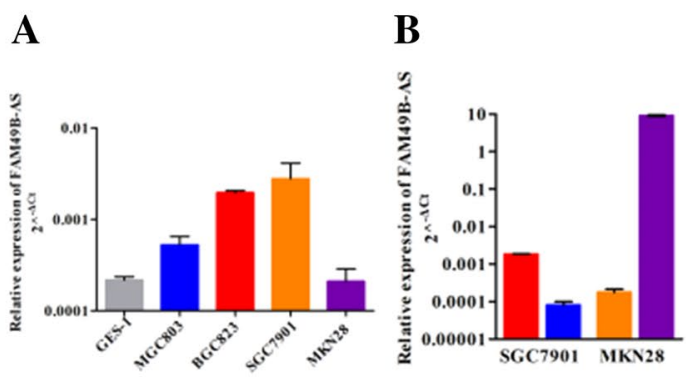

D

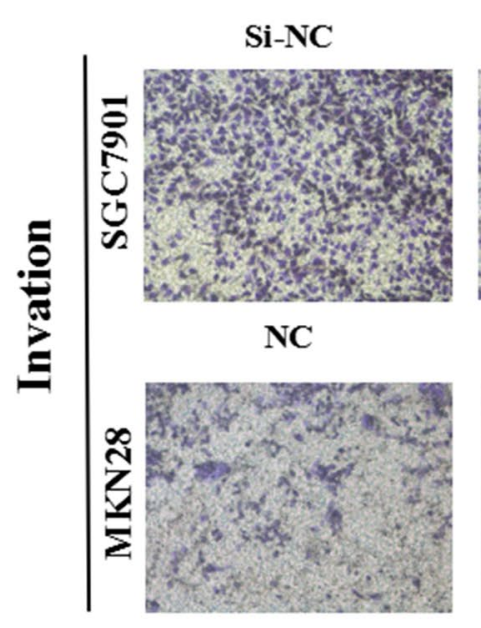

Si-FAM49B-AS

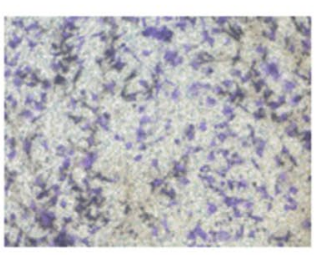

FAM49B-AS
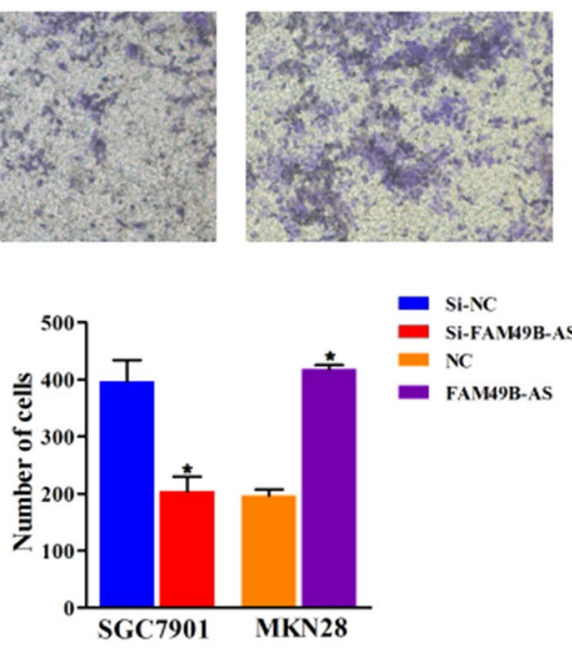

Fig. 5 Effect of plasma lncRNAs on GC cellular phenotypes. a qRTPCR detection of FAM49B-AS in GC cell lines (MGC803, BGC823, SGC7901, and MKN28) and GES-1. b SGC7901 cells were transfected with siRNAs/NC lentiviral vector of FAM49B-AS, and MKN28 cells were transfected with overexpression/NC of FAM49B-AS. Ectopic expression of FAM49B-AS in SGC7901 and MKN28 cells

would more accurately discriminate between cases and controls. The combination (three lncRNAs + CA242, three lncRNAs + CA724 and three IncRNAs + CA242 + CA724) outperformed the traditional biomarkers of CA242 or CA724 in terms of GC diagnosis. However, the combination had (three IncRNAs + CA242) lower AUC values $(\mathrm{AUC}=0.784)$ than the combination of these three lncRNAs $(A U C=0.818)$. We will enroll larger sample size to comprehensively detect the effect.
C

SGC7901

MKN28
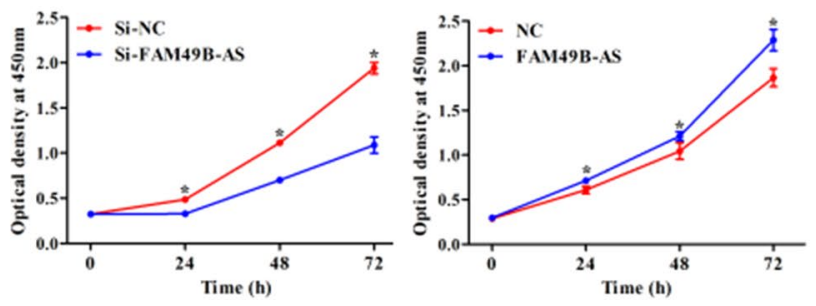

$\mathbf{E}$

Si-NC

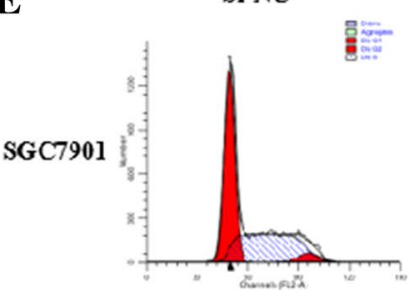

Si-FAM49B-AS

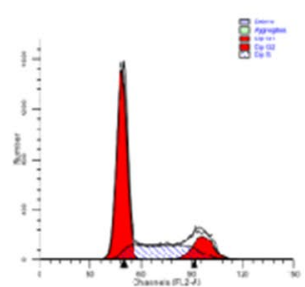

NC

FAM49B-AS
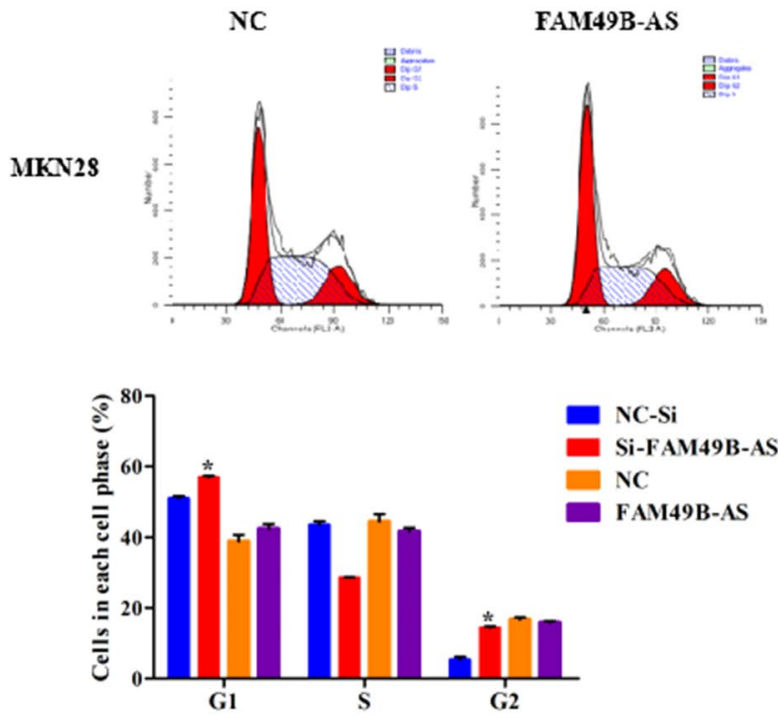

was detected using qRT-PCR. c Cell proliferation was measured by CCK8 assay. d Representative photographs of invasion assay for SGC7901 and MKN28 cells. The number of cells was counted. e Representative photographs of cell cycle analysis by flow cytometry. Results are presented as mean \pm SD. $* P<0.05$

Consistent with previous studies [29, 30], we investigated the origin of GC-related IncRNAs in plasma. Several studies have reported that plasma IncRNAs were primarily secreted from tumor cells, and would partly revert to normal levels after surgical treatment [17, 21]. Comparing lncRNA expressions in a subject's own preoperative and postoperative plasma can effectively follow their prognosis [31]. Therefore, we observed that the expression of these three IncRNAs was significantly reduced $10 \mathrm{~d}$ after surgery compared with 
before surgery, suggesting that these three lncRNAs could be used to monitor GC dynamics. Interestingly, FAM49B-AS levels could revert to healthy controls after surgery, indicating that FAM49B-AS may be more suitable for tracking GC. Extension of postoperative detection time is needed to further detect levels of GUSBP11 and CTDHUT. Meanwhile, candidate lncRNAs were significantly upregulated in GC cell lines and the corresponding culture medium compared with GES-1. In addition, these candidate IncRNAs could enter into culture medium of GC cell lines, increased with incubation time, indicating that they can be released from GC cells. Collectively, these findings provide indirect support for the GC-derived origin of lncRNAs.

Other studies have demonstrated that plasma lncRNAs are stable in RNase-rich blood [21, 32-34]. Similarly, these three candidate lncRNAs in plasma were almost stable even when treated with RNase A, prolonged room temperature incubation time or multiple freeze-thaw cycles. Recent studies have also reported that exosomes may incorporate lncRNAs and protect them from the RNase digestion in plasma, which might be the reason for the stability of IncRNAs in plasma. FAM49B has been widely reported to suppress pancreatic ductal adenocarcinoma by regulating mitochondrial function and integrity [35]. However, FAM49B's antisense transcript $F A M 49 B-A S$ remains poorly understood. Few studies have reported the role of GUSBP11 and CTDHUT in cancer. Recently, Cao et al. [36] reported that GUSBP11 was significantly associated with survival of patients with head and neck squamous cell carcinoma. We then chose FAM49B-AS to explore its cytological function according to the coding ability, transcript properties of candidate lncRNAs, and the capability of tracking disease. We found that the knockdown of FAM49B-AS inhibited the proliferation and invasion of GC cells, whereas opposite findings were observed with overexpression of FAM49B-AS.

The present study used genome-wide plasma lncRNAs profiles to identify promising lncRNA biomarkers for GC. In the two-stage validation, we first identified that plasma FAM49B-AS, GUSBP11, and CTDHUT were significantly overexpressed in GC. Then we systematically confirmed that the combined use of the three plasma lncRNAs could provide a more effective diagnostic power for GC diagnosis. Since we studied the effect of FAM49B-AS on GC cells, we believe that the function of all three candidate lncRNAs should be explored in further studies. Moreover, it is well known that Helicobacter pylori infection is one of the important factors for GC occurrence [37]; however, we lack data for Helicobacter pylori infection from subjects, which might have limited the ability to comprehensively evaluate the diagnostic power of these three lncRNAs for GC.

In conclusion, we systematically characterized three novel GC-related lncRNAs (FAM49B-AS, GUSBP11, and $C T D H U T$ ) in plasma as promising novel noninvasive biomarkers in GC diagnosis using plasma lncRNAs expression profiling arrays.

Acknowledgements This study was supported by National Natural Science Foundation of China (81473049, 81773538 and 81773539), Jiangsu Provincial Science and Technology Innovation Team, Jiangsu Provincial Postdoctoral Science Foundation funded project (1501081C), China Postdoctoral Science Foundation funded project (2015M580449), Collaborative Innovation Center For Cancer Personalized Medicine, and the Priority Academic Program Development of Jiangsu Higher Education Institutions (Public Health and Preventive Medicine).

Author contributions $\mathrm{ZZ}, \mathrm{QF}, \mathrm{DM}$ and $\mathrm{WM}$ designed the research. WW, ZQ, TG and ZG recruited samples. ZR, LJ, LJ, LS, WX, LM and $\mathrm{CH}$ contributed reagents/materials/analysis tools. ZR, LJ and LJ wrote the manuscript. All authors reviewed the manuscript.

\section{Compliance with ethical standards}

Conflict of interest The authors declare that they have no conflict of interest.

Human rights statement and informed consent The research was approved by the Ethics Committee of Nanjing Medical University. Informed consent was obtained from all subjects before they were included in this study.

\section{References}

1. Siegel RL, Miller KD, Jemal A. Cancer statistics, 2018. CA Cancer J Clin. 2018;68:7-30.

2. Torre LA, Bray F, Siegel RL, Ferlay J, Lortet-Tieulent J, Jemal A. Global cancer statistics, 2012. CA Cancer J Clin. 2015;65:87-108.

3. Chen W, Zheng R, Baade PD, Zhang S, Zeng H, Bray F, et al. Cancer statistics in China, 2015. CA Cancer J Clin. 2016;66:115-32.

4. Kunz PL, Gubens M, Fisher GA, Ford JM, Lichtensztajn DY, Clarke CA. Long-term survivors of gastric cancer: a California population-based study. J Clin Oncol. 2012;30:3507-15.

5. Camargo MC, Kim WH, Chiaravalli AM, Kim KM, Corvalan AH, Matsuo K, et al. Improved survival of gastric cancer with tumour Epstein-Barr virus positivity: an international pooled analysis. Gut. 2014;63:236-43.

6. Zhou YC, Zhao HJ, Shen LZ. Preoperative serum CEA and CA19-9 in gastric cancer - a single tertiary hospital study of 1,075 cases. Asian Pac J Cancer Prev. 2015;16:2685-91.

7. Tian SB, Yu JC, Kang WM, Ma ZQ, Ye X, Cao ZJ, et al. Combined detection of CEA, CA 19-9, CA 242 and CA 50 in the diagnosis and prognosis of resectable gastric cancer. Asian Pac J Cancer Prev. 2014;15:6295-300.

8. Mattar R, Alves de Andrade CR, DiFavero GM, Gama-Rodrigues JJ, Laudanna AA. Preoperative serum levels of CA 72-4, CEA, CA 19-9, and alpha-fetoprotein in patients with gastric cancer. Rev Hosp Clin Fac Med Sao Paulo. 2002;57:89-92.

9. Zhang K, Shi H, Xi H, Wu X, Cui J, Gao Y, et al. Genome-wide lncRNA microarray profiling identifies novel circulating lncRNAs for detection of gastric cancer. Theranostics. 2017;7:213-27.

10. Mishra PJ. Non-coding RNAs as clinical biomarkers for cancer diagnosis and prognosis. Expert Rev Mol Diagn. 2014;14(8):917-9 
11. Li PF, Chen SC, Xia T, Jiang XM, Shao YF, Xiao BX, et al. Non-coding RNAs and gastric cancer. World J Gastroenterol. 2014;20:5411-9.

12. Shi T, Gao G, Cao Y. Long noncoding RNAs as novel biomarkers have a promising future in cancer diagnostics. Dis Markers. 2016;9085195:10.

13. Zhang H, Chen Z, Wang X, Huang Z, He Z, Chen Y. Long non-coding RNA: a new player in cancer. J Hematol Oncol. 2013;6:1756-8722.

14. Qiu MT, Hu JW, Yin R, Xu L. Long noncoding RNA: an emerging paradigm of cancer research. Tumour Biol. 2013;34:613-20.

15. Ren S, Wang F, Shen J, Sun Y, Xu W, Lu J, et al. Long non-coding RNA metastasis associated in lung adenocarcinoma transcript 1 derived miniRNA as a novel plasma-based biomarker for diagnosing prostate cancer. Eur J Cancer. 2013;49:2949-59.

16. Amorim M, Salta S, Henrique R, Jeronimo C. Decoding the usefulness of non-coding RNAs as breast cancer markers. J Transl Med. 2016;14:016-1025.

17. Tong YS, Wang XW, Zhou XL, Liu ZH, Yang TX, Shi WH, et al. Identification of the long non-coding RNA POU3F3 in plasma as a novel biomarker for diagnosis of esophageal squamous cell carcinoma. Mol Cancer. 2015;14:1476-4598.

18. Li Q, Shao Y, Zhang X, Zheng T, Miao M, Qin L, et al. Plasma long noncoding RNA protected by exosomes as a potential stable biomarker for gastric cancer. Tumour Biol. 2015;36:2007-12.

19. Pang Q, Ge J, Shao Y, Sun W, Song H, Xia T, et al. Increased expression of long intergenic non-coding RNA LINC00152 in gastric cancer and its clinical significance. Tumour Biol. 2014;35:5441-7

20. Song H, Sun W, Ye G, Ding X, Liu Z, Zhang S, et al. Long noncoding RNA expression profile in human gastric cancer and its clinical significances. J Transl Med. 2013;11:1479-5876.

21. Zhou X, Yin C, Dang Y, Ye F, Zhang G. Identification of the long non-coding RNA H19 in plasma as a novel biomarker for diagnosis of gastric cancer. Sci Rep. 2015;5:11516.

22. Liu J, Wang J, Song Y, Ma B, Luo J, Ni Z, et al. A panel consisting of three novel circulating lncRNAs, is it a predictive tool for gastric cancer? J Cell Mol Med. 2018;26:13640.

23. Gong C, Li Z, Ramanujan K, Clay I, Zhang Y, Lemire-Brachat $\mathrm{S}$, et al. A long non-coding RNA, LncMyoD, regulates skeletal muscle differentiation by blocking IMP2-mediated mRNA translation. Dev Cell. 2015;34:181-91.

24. Duraes C, Almeida GM, Seruca R, Oliveira C, Carneiro F. Biomarkers for gastric cancer: prognostic, predictive or targets of therapy? Virchows Arch. 2014;464:367-78.

25. Van Cutsem E, Sagaert X, Topal B, Haustermans K, Prenen H. Gastric cancer. Lancet. 2016;388:2654-64.
26. Arita T, Ichikawa D, Konishi H, Komatsu S, Shiozaki A, Shoda $\mathrm{K}$, et al. Circulating long non-coding RNAs in plasma of patients with gastric cancer. Anticancer Res. 2013;33:3185-93.

27. Liu Z, Shao Y, Tan L, Shi H, Chen S, Guo J. Clinical significance of the low expression of FER1L4 in gastric cancer patients. Tumour Biol. 2014;35:9613-7.

28. Shao Y, Ye M, Jiang X, Sun W, Ding X, Liu Z, et al. Gastric juice long noncoding RNA used as a tumor marker for screening gastric cancer. Cancer. 2014;120:3320-8.

29. Toiyama Y, Takahashi M, Hur K, Nagasaka T, Tanaka K, Inoue $\mathrm{Y}$, et al. Serum miR-21 as a diagnostic and prognostic biomarker in colorectal cancer. J Natl Cancer Inst. 2013;105:849-59.

30. Bhan A, Soleimani M, Mandal SS. Long noncoding RNA and cancer: a new paradigm. Cancer Res. 2017;77:3965-81.

31. Hotta T, Takifuji K, Yokoyama S, Matsuda K, Oku Y, Nasu T, et al. Impact of the post/preoperative serum CEA ratio on the survival of patients with rectal cancer. Surg Today. 2014;44:2106-15.

32. Lorenzen JM, Schauerte C, Kielstein JT, Hubner A, Martino F, Fiedler $\mathbf{J}$, et al. Circulating long noncoding RNATapSaki is a predictor of mortality in critically ill patients with acute kidney injury. Clin Chem. 2015;61:191-201.

33. Dong L, Qi P, Xu MD, Ni SJ, Huang D, Xu QH, et al. Circulating CUDR, LSINCT-5 and PTENP1 long noncoding RNAs in sera distinguish patients with gastric cancer from healthy controls. Int J Cancer. 2015;137:1128-35.

34. Isin M, Ozgur E, Cetin G, Erten N, Aktan M, Gezer U, et al. Investigation of circulating lncRNAs in B-cell neoplasms. Clin Chim Acta. 2014;431:255-9.

35. Chattaragada MS, Riganti C, Sassoe M, Principe M, Santamorena MM, Roux C, et al. FAM49B, a novel regulator of mitochondrial function and integrity that suppresses tumor metastasis. Oncogene. 2018;37:697-709.

36. Cao W, Liu JN, Liu Z, Wang X, Han ZG, Ji T, et al. A threelncRNA signature derived from the Atlas of ncRNA in cancer (TANRIC) database predicts the survival of patients with head and neck squamous cell carcinoma. Oral Oncol. 2017;65:94-101.

37. Wang F, Meng W, Wang B, Qiao L. Helicobacter pyloriinduced gastric inflammation and gastric cancer. Cancer Lett. 2014;345:196-202.

Publisher's Note Springer Nature remains neutral with regard to jurisdictional claims in published maps and institutional affiliations.

\section{Affiliations}

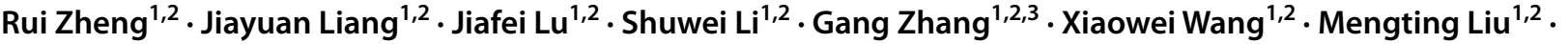 Weizhi Wang ${ }^{4}$. Haiyan Chu ${ }^{1,2}$. Guoquan Tao ${ }^{5}$. Qinghong Zhao ${ }^{6}$. Meilin Wang ${ }^{1,2,7} \cdot$ Mulong Du $^{1,8} \cdot$ Fulin Qiang $^{9}$. Zhengdong Zhang ${ }^{1,2,7}$}

1 Department of Environmental Genomics, Jiangsu Key Laboratory of Cancer Biomarkers, Prevention and Treatment, Collaborative Innovation Center for Cancer Personalized Medicine, Nanjing Medical University, Nanjing, China

2 Department of Genetic Toxicology, The Key Laboratory of Modern Toxicology of Ministry of Education, School of Public Health, Nanjing Medical University, Nanjing, China
3 Department of Neurology, The Affiliated Children's Hospital of Nanjing Medical University, Nanjing, China

4 Department of General Surgery, The First Affiliated Hospital of Nanjing Medical University, Nanjing, China

5 Department of General Surgery, The Affiliated Huaian No.1 People's Hospital of Nanjing Medical University, Huai'an, China 
6 Department of General Surgery, The Second Affiliated Hospital of Nanjing Medical University, Nanjing, China

7 Department of Environmental Genomics, Center for Global Health, School of Public Health, Nanjing Medical University, 101 Longmian Avenue, Jiangning District, Nanjing 211166, China
8 Department of Biostatistics, Center for Global Health, School of Public Health, Nanjing Medical University, 101 Longmian Avenue, Jiangning District, Nanjing 211166, China

9 Department of Core Laboratory, Nantong Tumor Hospital, Nantong, China 